\title{
Spatial Discretization and Approximation of Distributed Feedback Loops and Inputs
}

\author{
Yakar Kannai $^{1}$, Matei Kelemen ${ }^{2}$, Isaac Horowitz ${ }^{3}$
}

1. Dept. of Mathematics, The Weizmann Institute of Science, Rehovot 76100, Israel

2. Dept. of Applied Mathematics, Twente University, 7500 AE Enschede, The Netherlands

3. Dept. of Electrical Engineering, University of California, Davis, CA 95616

\begin{abstract}
The problem of approximating distributed feedback loops and inputs by discrete (in space) ones is studied in some detail. The main result, based on integral equations, gives sufficient conditions on the plant, input and compensator enabling such an approximation. The result is illustrated by two examples.
\end{abstract}

\section{Introduction}

It was recognized since a long time ago (see for instance [1] and [2]) that a practical approach to the design of distributed parameter feedback systems requires a finite number of locations where measurement and control should take place. It was proved in [3] that, under certain circumstances, it is possible to achieve desired performances in a finite number of points, by processing a finite number of measurements. One would like, however, to obtain satisfactory performance over all of the space range, by using only a finite (possibly large) number of controls. Our goal in this article, motivated by design reasons, is to see what happens in closed loop and in the frequency domain (of the time variable). More precisely, let us assume that there is a distributed system with a certain output (depending on the space variable as well), due to a distributed (in space) input and a distributed feedback loop. In section 2 we give conditions assuring that the same output can be arbitrarily closely approximated, by using a finite number of point feedback loops and inputs. The point feedback loops and inputs have to approximate closely enough, in a weak (distribution) sense, the distributed feedback (respectively input).

Our proof is based on the Fredholm-Hilbert idea for solving integral equations (compare also [4], section 4.16). Our model differs from the one considered in a recent paper [5]. There, a fixed irrational matrix describing a plant is approximated by a rational matrix, whereas we are interested in approximating feedback loops continuously distributed in space by discretely distributed loops.

The result in this article suggests a way for the "approximate" implementation of continuous (in space) feedback loops. Note that, while continuous feedback loops are, generally, easier to design than discrete (in space) ones (see for instancc [5]), they are not realistically implementable.

\section{Spatial Discretization of Distributed}

$$
\text { Feedback Loops and Inputs }
$$

Let us consider a system described by a linear PDE

$$
(\mathcal{P} y)(x, t)=(d+u)(x, t) .
$$

Here $x \in[a, b]$ is the space variable, $t>0$ is the time variable, $y$ is the output, $d$ is the input, $u$ is the "feedback" input, and $\mathcal{P}$ is a linear partial differential operator (in $\frac{\partial}{\partial x}$ and $\frac{\partial}{\partial t}$ ), with coefficients independent of $t$.
We shall consider zero initial conditions and boundary conditions. The boundary conditions are so as to ensure the well-posedness of the problem of finding $y$, when $u$ and $d$ are given functions belonging to appropriate classes. We embed the above plant in a feedback loop, by setting

$$
u(x, t)=(\mathcal{G} y)(x, t),
$$

where the compensator $\mathcal{G}$ is a linear, time invariant operator (pseudodifferential) in $\frac{\partial}{\partial t}$, whose coefficients might depend on $x$.

By considering the Laplace transform in (time) of (1) and (2) we obtain a family (parametrized by $s$ ) of linear ODE's with boundary conditions

$$
(P+G) Y=D .
$$

Here $P$, the Laplace transform of $\mathcal{P}$, is a linear differential operator (in $\frac{d}{d x}$ ), with coefficients depending on $x$ and $s$, while $G, D, Y$ are functions representing the Laplace transform of $\mathcal{G}, d, y$ respectively. (The zero boundary conditions carry over to $Y$ and assure uniqueness.) Assume now that $G$ and $D$ are continuous functions of $x$. Then, we can approximate them (in distribution sense) by

$$
\begin{aligned}
& G_{n}(x, s)=\gamma_{n} \sum_{i=1}^{n} G\left(x_{i}, s\right) \delta_{x_{i}}(x) \\
& D_{m}(x, s)=\gamma_{m} \sum_{j=1}^{m} D\left(z_{j}, s\right) \delta_{z_{j}}(x),
\end{aligned}
$$

whete $\gamma_{n}=\frac{b-a}{n}, x_{i}=a+i \gamma_{n}, \gamma_{m}=\frac{b-a}{m}, z_{j}=a+j \gamma_{m}$, $i=1, \ldots, n, j=1, \ldots, n, \delta$ is the Dirac impulse.

Let us substitute the expressions ( 3 ) in the equation ( $\left.1^{\prime}\right)$. Then, by a direct computation (using superposition) we see that the solution $Y_{n, m}$ of the resulting equation satisfies, formally, the following relation

$$
\begin{aligned}
Y_{n, m}(x)= & -\gamma_{n} \sum_{i=1}^{n} \Phi\left(x, x_{i}\right) G\left(x_{i}\right) Y_{n, m}\left(x_{i}\right) \\
& +\gamma_{m} \sum_{j=1}^{m} \Phi\left(x, z_{j}\right) D\left(z_{j}\right) .
\end{aligned}
$$

Here $\Phi\left(x, x_{i}\right)$ is the Green function corresponding to (1') with $G=0$ and the given (zero) boundary conditions, i.e.

$$
P \Phi\left(x, x_{i}\right)=\delta_{x_{i}}(x)
$$

and we have dropped the argument $s$, as we shall from now on. 
A natural question is whether $Y_{m, n}$ approaches the solution $Y$ of (3.1') (corresponding to $G$ and $D$ ), when $n$ an $m$ tend to infinity (i.e. $G_{n}$ and $D_{n}$ tend to $G$ and $D$ respectively). Set $\Omega_{\sigma}=\{s \mid$ Res $>\sigma\}$ ).

THEOREM: Assume that there is a $\sigma_{0}$ such that

a) $\Phi, G, D$ are bounded, uniform continuous functions, $\Phi$ on $[a, b]^{2} \times \Omega_{\sigma_{0}}, G$ and $D$ on $[a, b] \times \Omega_{\sigma_{0}}$, and analytic on $\Omega_{\sigma_{0}}$ for any fixed $(x, z) \in[a, b]^{2}$, respectively $x \in[a, b]$

b)

$$
\lim _{s \rightarrow \infty} \Phi(x, z) G(z)=0
$$

on $\Omega_{\sigma_{0}}$, uniformly in $(x, z) \in[a, b]^{2}$.

Then $Y_{n, m}$ are continuous on $[a, b] \times \Omega_{\sigma_{0}}$, and there exists a $\sigma_{1} \geq \sigma_{0}$ such that

$$
\lim _{\substack{m \rightarrow \infty \\ n \rightarrow \infty}} Y_{n, m}(x)=Y(x)
$$

uniformly in $(x, s) \in[a, b] \times \Omega_{\sigma_{1}}$.

The essential part in the proof is a result on the convergence of certain approximate solutions of a Fredholm integral equation (compare [4] ). The relevant integral equation is

$$
Y(x)+\int_{a}^{b} \Phi(x, z) G(z) Y(z) d z=\int_{a}^{b} \Phi(x, z) D(z) d z,
$$

compare equation (1').

\section{Examples}

Heat and (damped) Wave equations

Let us begin with the heat equation, i.e. the operator $\mathcal{P}$ is described by

$$
\begin{aligned}
& \frac{\partial y}{\partial t}-\frac{\partial^{2} y}{\partial x^{2}}=d(x, t), x \in(0, \pi), t>0 \\
& y(x, 0)=0, x \in[0, \pi] \\
& y(0, t)=y(\pi, t)=0 .
\end{aligned}
$$

The equation of the plant (obtained by considering the Laplace transform in the time variable), i.e. the operator $P$ is given by

$$
\begin{aligned}
& s Y-\frac{\partial^{2} Y}{\partial x^{2}}=D(x, s), x \in(0, \pi), t>0 \\
& Y(0, s)=Y(\pi, s)=0 .
\end{aligned}
$$

Then, the Green function is (see also [5])

$$
\Phi\left(x, x_{i}, s\right)=\frac{1}{\sqrt{s}} \frac{\left(e^{\sqrt{s}\left(\pi-x_{i}\right)}-e^{-\sqrt{s}\left(\pi-x_{i}\right)}\right)\left(e^{\sqrt{s} x}-e^{-\sqrt{s} x}\right)}{2\left(e^{\sqrt{s} \pi}-e^{-\sqrt{s} \pi}\right)},
$$

if $0 \leq x \leq x_{i}$; for $x_{i} \leq x \leq \pi$ the solution is given by the same formula but with $x$ and $\pi-x_{i}$ replaced by $\pi-x$ respectively $x_{i}$.

We claim that $\sigma_{0}$ (from the theorem) can be chosen as any number greater than -1 (see the Fourier expansion in [5]). We shall prove only the uniform continuity on the unbounded domain $\Omega_{\sigma_{0}}$. This is a consequence of the fact that $\Phi$, when $s$ tends to infinity, goes to zero as $\frac{1}{\sqrt{s}}$. Hence $\Phi$ satisfies the requirement a) of the theorem. Thus, the theorem is applicable for the heat equation plant.
That is, assume that the compensator $G$, which is a continuous function of $(x, s)$, is designed in such a way that the output $Y$ of the closed loop system (1') with zero boundary conditions

$$
\left(s-\frac{\partial^{2}}{\partial x^{2}}+G\right) Y=D
$$

satisfies certain specifications when the input $D$ is a given in a class of continuous function of $(x, s)$. ( $G$ and $D$ have to satisfy some "technical" assumptions too, stated in the hypotheses of the theorem.) Then, if we replace $G$ and $D$ with the discrete (i.e. more realistically implementable) approximates $G_{n}$ and $D_{m}($ see $(3))$

$$
\begin{aligned}
& G_{n}(x, s)=\frac{\pi}{n} \sum_{i=1}^{n} G\left(\frac{i \pi}{n}, s\right) \delta_{\frac{i \pi}{n}}(x) \\
& D_{m}(x, s)=\frac{\pi}{m} \sum_{j=1}^{m} D\left(\frac{j \pi}{m}, s\right) \delta_{\frac{j \pi}{m}}(x),
\end{aligned}
$$

the output will be a continuous function $Y_{n, m}$. Our theorem shows that if $n$ and $m$ are large enough, then $Y_{n, m}$ can be made arbitrarily close (in norm) to $Y$.

We consider next the (damped) wave equation

$$
\begin{aligned}
& \frac{\partial^{2} y}{\partial t^{2}}-\frac{\partial^{2} y}{\partial x^{2}}+2 \epsilon \frac{\partial y}{\partial t}=u(x, t), x \in(0, \pi), t>0 \\
& \frac{\partial y}{\partial t}(x, 0)=0, x \in[0, \pi] \\
& y(x, 0)=0, x \in[0, \pi] \\
& y(0, t)=y(\pi, t)=0 .
\end{aligned}
$$

Here $0 \leq \epsilon \leq 1$, and $\Phi$ is given by the same formula (7) as in the case of the heat equation, but with $s$ replaced by $s^{2}+2 \epsilon s$. Hence, the theorem is applicable for this plant too, but with $\sigma_{0}>-\epsilon$.

REMARK For both stability and design reasons, one would like to have $\sigma_{1}<0$. This requirement can be satisfied, with no constraint on the magnitude of (a properly chosen) $G$, for the heat equation, and for the other previous examples, provided $\epsilon>0$. When $\epsilon=0$, the (undamped) wave and beam equations cannot meet the above requirement, since $\sigma_{0}$ is necessarily greater than zero.

\section{$\underline{\text { References }}$}

[1] J.R. Melcher, An experiment to stabilize an electromechanical continuum, IEEE Trans. AC, 10 (1965), pp. 466-469.

[2] M. Athans, Toward a practical theory of distributed parameter systems, IEEE Trans. AC, 15 (1970), pp. 245-247.

[3] S.A. Pohjolainen, Robust multivariable PI- controller for infinite dimensional systems, IEEE Trans. AC, 27 (1982), pp. 17-30.

[4] C. Baker, The numerical treatment of integral equations: Clarendon Press, Oxford, 1977.

[5] M. Kelemen, Y. Kannai and I. Horowitz, One point feedback approach to distributed linear systems, Int. J. Control, 49 (1989) pp. 969-980. 